\title{
Explorar la movilidad estudiantil: Facultad de Filosofía y Letras de la UNAM
}

\author{
Luis Alberto Sandoval Rosete y Vianey Santiago Munilla
}

\begin{abstract}
Resumen
El presente artículo da cuenta de los principales rasgos de la movilidad estudiantil en la Facultad de Filosofía y Letras de la Universidad Nacional Autónoma de México (UNAM). La metodología que se empleó fue la descriptiva, los datos fueron proporcionados por el Área de Movilidad e Intercambio Académico (bases de datos). Los resultados mostraron que un pequeño porcentaje de estudiantes de la entidad son los que realizan movilidad (menos de 1\% de la población estudiantil total), la mayoría son mujeres. La principal preferencia es la movilidad internacional, sobre todo a países de la Unión Europea, Asia Oriental y América del Norte; sin embargo, también se eligen países de habla hispana. Por último, se presentan algunas ventajas y desventajas de la movilidad estudiantil a nivel superior.
\end{abstract}

Palabras clave: internacionalización, movilidad estudiantil, educación superior, cooperación educativa, política educativa.

\section{Exploring student mobility: Faculty of Philosophy and Letters of the UNAM}

\begin{abstract}
This article gives an account of the main features of student mobility in the Faculty of Philosophy and Letters of the National Autonomous University of Mexico (UNAM). The methodology used was descriptive, the data was provided by the Mobility and Academic Exchange Area (databases). The results showed that a small percentage of students of the entity are those who perform mobility (less than $1 \%$ of the total student population), most of them are women. The main preference is international mobility, especially to countries of the European Union, East Asia and North America; however, Spanish-speaking countries are also chosen. Finally, we discuss some advantages and disadvantages of student mobility at a higher level.
\end{abstract}

Keywords: internationalization, student mobility, higher education, educational cooperation, educational policy.

Dol: http://doi.org/10.22201/codeic.16076079e.2019.v20n5.a9 


\section{Luis Alberto Sandoval Rosete}

Estudió la preparatoria en el Colegio de Bachilleres, en el cual obtuvo un certificado de técnico en programación, actualmente cursa el séptimo semestre de la licenciatura en Pedagogía en la Facultad de Filosofía y Letras de la UNAM.

Algunos de sus temas de interés son la psicología educativa, la investigación educativa, el uso y desarrollo de las tecnologías de la información y comunicación en la educación, así como temas relacionados con la interculturalidad.

\section{Vianey Santiago Munilla}

vianeysmunilla@gmail.com

Estudió en la Escuela Nacional Preparatoria número 7. Actualmente es estudiante de séptimo semestre de la licenciatura en Pedagogía en la Facultad de Filosofía y Letras de la UNAM.

Se interesa por la investigación educativa, las tecnologías de la información y la comunicación aplicadas a la educación, la educación no formal, el diseño curricular, la interculturalidad, y la política educativa. 


\section{Introducción}

La movilidad estudiantil se refiere esencialmente a un proceso diseñado para facilitar la estancia de los alumnos en otras instituciones educativas de su mismo país o del extranjero. Ésta ha tenido un mayor desarrollo en la educación superior y su principal ventaja es dar a los alumnos una visión más amplia, representando una gran oportunidad para enriquecer su perfil profesional. Asimismo, la internacionalización de la educación superior se ha establecido como una estrategia para mejorar la calidad de las instituciones universitarias y como herramienta para el desarrollo económico, social y educativo de cada país.

Los estudiantes que realizan movilidad pueden hacerlo a través de distintas modalidades: al cursar asignaturas aisladas, durante un ciclo completo, semestre o año, o mediante prácticas profesionales, o estancias de investigación. Todo en el marco de las disposiciones y normas de la institución de origen y de la de destino.

El objetivo de este artículo es dar cuenta de los principales rasgos de la movilidad estudiantil dentro de la Facultad de Filosofía y Letras (FFyL) de la UNAM: número y porcentaje de estudiantes, los países que eligen y las principales instituciones a las que se van.

El artículo se encuentra estructurado en cinco apartados: el primero, se centra en la internacionalización de la educación superior; el segundo aborda la internacionalización en el contexto mexicano y específicamente en la UNAM; el tercero, da cuenta de la movilidad en la Facultad de Filosofía y Letras; el cuarto apartado muestra algunas de las implicaciones de realizar la movilidad y en el último se presentan las consideraciones finales.

\section{Internacionalización de la educación superior}

Aunque hay una gran cantidad de antecedentes de internacionalización en la educación superior, este apartado se centra en el contexto europeo con el Proceso de Bolonia en 1999, el cual estableció diversas políticas con la intención de mejorar la educación y la formación de los estudiantes, abordando la educación superior desde un ámbito científico multidisciplinar que permitió la conexión de los educandos con otros entornos sociales en constante cambio (Fraile, 2006; Rodríguez, 2018).

Dicho proceso permitió la creación del Espacio Europeo de Educación Superior (EEES) que se ha constituido a partir de:

1. Un sistema común de créditos que facilita la movilidad de los estudiantes: sistema de equivalencias y de reconocimiento de los estudios cursados en otros países. 
Fotografía 1. Elaboración propia.
2. Un sistema de titulaciones comparable para promover la competitividad internacional de los sistemas educativos superiores.

3. Promoción de la movilidad.

4. Impulso a la cooperación europea para garantizar metodologías educativas comparables.

5. Fomento de la dimensión europea de la educación superior (Fraile, 2006).

Estos puntos, con el paso de los años, se fueron expandiendo a otros países del mundo, lo cual ha incrementado las oportunidades de movilidad a muchos estudiantes en diferentes territorios. Ejemplo de ello fue la creación de la Red de Universidades Regionales de Latinoamérica (Red Urel) en la segunda mitad de los años noventa, con el objetivo de contribuir a la integración educativa, cultural y académica de dichas universidades (Fraile, 2006).

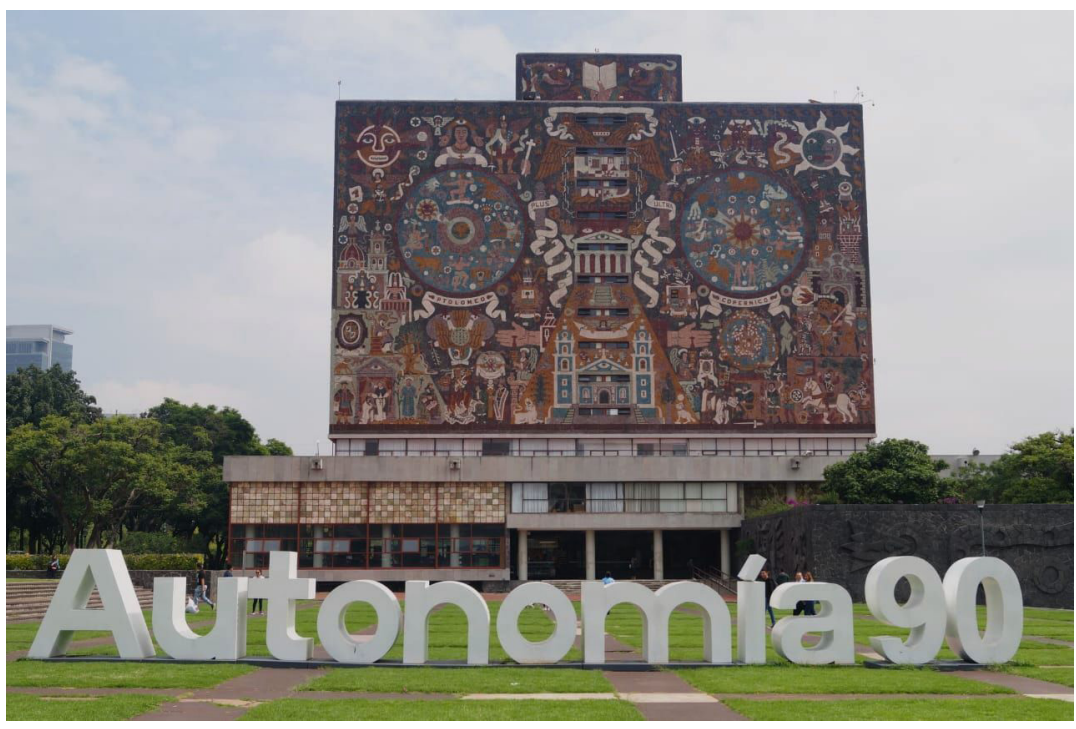

\section{Internacionalización de la educación superior en México}

La internacionalización de la educación superior en México tomó fuerza en los últimos años de la década de los ochenta; sin embargo, tiene antecedentes más remotos. Desde la década de los sesenta México tenía algunos acuerdos para la movilización internacional, con la principal intención de obtener un intercambio cultural. Para la década de los noventa, la internacionalización de la educación superior pasó a formar parte de la agenda política, de manera que, las universidades tomaron este proceso como un "eje estratégico de desarrollo institucional para alcanzar mayor calidad educativa y enfrentar los desafíos del siglo xxl" (Corbella y Elías, 2018, p. 122).

En 2004, resalta la constitución del Espacio Común de Educación Superior (ECOES), el cual se integró a iniciativa de la Universidad Nacional Autónoma de México, del Instituto Politécnico Nacional (IPN) y la Universidad Autónoma 
Metropolitana (UAM). Actualmente está constituido por 43 Instituciones de Educación Superior (ECOES, 2019).

De 2007 a 2012, la política educativa tuvo como base el Programa Sectorial de Educación, en el cual se incluyó la internacionalización de la educación superior para incrementar la calidad en la educación. Lo anterior alentó los acuerdos de colaboración entre instituciones mexicanas y extranjeras, favoreció el establecimiento de redes de cooperación académica, e impulsó el reconocimiento de créditos, el intercambio académico y el otorgamiento de títulos conjuntos (SEP, 2007). Algunas de las dependencias gubernamentales que gestionan los acuerdos y convenios que permiten el cumplimiento de estos objetivos son la Secretaría de Educación Pública (SEP), la Secretaría de Relaciones Exteriores (SRE) y el Consejo Nacional de Ciencia y Tecnología (CONACYT).

Por último, en el Programa Sectorial de Educación 2013-2018, se hace referencia a que la educación superior promueve la generación de conocimiento en las áreas de ciencia y tecnología por medio de la investigación y la cooperación nacional e internacional, principalmente en el posgrado, permitiendo así abrir el paso al camino del desarrollo del país (SEP, 2013).

\section{Movilidad en la Universidad Nacional Autónoma de México}

La UNAM, a lo largo de la historia, ha contado con diferentes oficinas de internacionalización, las cuales se han ido modificando con los nuevos contextos tanto nacionales como internacionales.

Actualmente la que prevalece y coordina todos los asuntos de movilidad e intercambio estudiantil es la Dirección General de Cooperación e

Fotografía 2. Elaboración propia. Internacionalización (UNAM-DGECI), encargada de "fomentar y operar programas de intercambio estudiantil internacional de nivel licenciatura, así como de

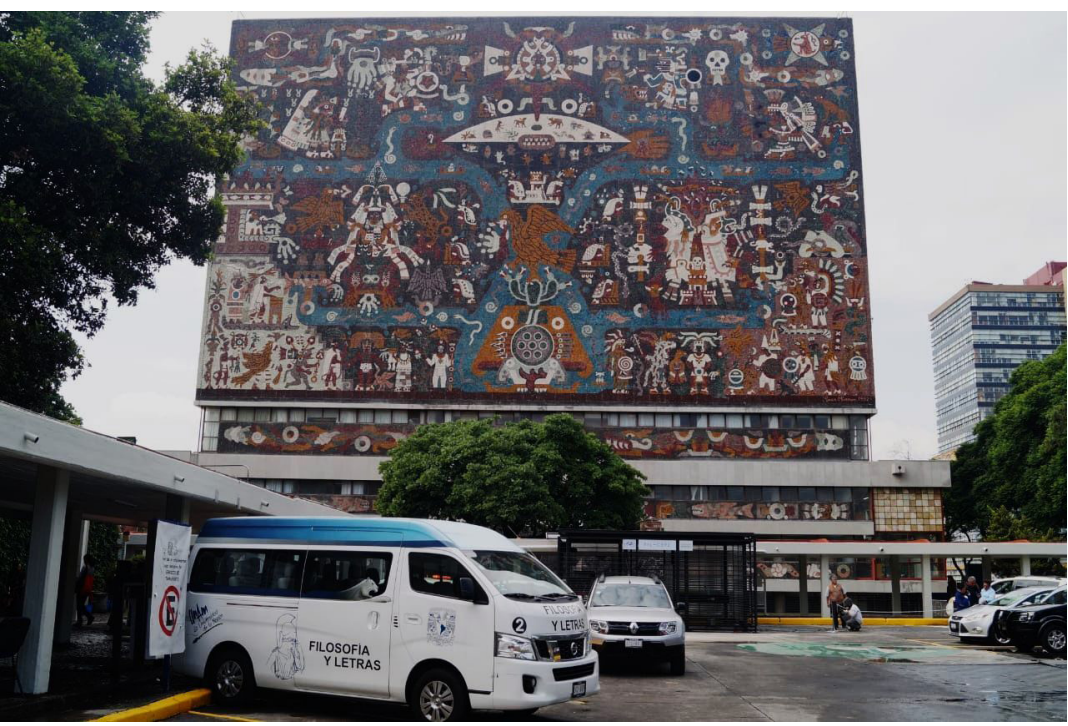
la suscripción de convenios de colaboración académica con instituciones de educación superior de otros países" (UNAM-DGECl, 2018). Además, apoya a la planeación y desarrollo de estrategias que fortalecen la cooperación académica internacional con otras instituciones de educación superior.

La DGECI, también proporciona orientación y atención a los estudiantes y académicos, con el objetivo primordial de brindar información oportuna de acuerdo a las necesidades de la comunidad de la UNAM, referente a la internacionalización y movilidad estudiantil, así como a los estudiantes extranjeros a los que les interese realizar una estancia en la Universidad. 
Tabla 1. Porcentaje de estudiantes que realizaron movilidad internacional por licenciatura (semestre 2017-1).

Fuente: elaboración propia a partir de los datos proporcionados por el Área de Movilidad e Intercambio Académico de la Facultad de Filosofía y Letras de la UNAM.
Es relevante mencionar que, para el mes de enero del 2019,480 estudiantes cursaron un semestre en universidades de 29 países diferentes (UNAM-DGCS, 2018). Sin embargo, no todos los estudiantes pueden participar en un programa de movilidad, pues deben cumplir una serie de requisitos y obligaciones.

\section{La movilidad estudiantil en la Facultad de Filosofía y Letras de la UNAM}

En la FFyL se imparten quince licenciaturas y la movilidad estudiantil tiene dos vertientes: la internacional y la nacional. Con base en información proporcionada por la oficina de Programas Estudiantiles-Área de Movilidad e Intercambio Académico de la FFyL en este apartado se analiza la participación de los estudiantes en ambos programas de movilidad durante el año escolar 2017 _ segundo semestre de 2016 (2017-1) y primer semestre del 2017 (2017-2)_y se identifican las universidades a las que los alumnos de la Facultad prefieren ir a estudiar. La información se obtuvo de diversas bases de datos proporcionadas por el Área de Movilidad e Intercambio Académico de la FFyL.

\section{Movilidad internacional}

En el semestre 2017-1 (de agosto a diciembre 2016), se fueron de movilidad internacional 29 estudiantes, los cuales representan $0.37 \%$ de la población estudiantil total. Como muestra la tabla 1 las licenciaturas que tuvieron mayor presencia en este período fueron Literatura Dramática y Teatro con 17.2\% de estudiantes y Geografía con 13.7\%.

\begin{tabular}{ccccc} 
No. & Licenciatura & Total & Femenino & Masculino \\
\hline 1 & Literatura Dramática y Teatro & $17.2 \%$ & $60 \%$ & $40 \%$ \\
2 & Geografía & $13.7 \%$ & $50 \%$ & $50 \%$ \\
3 & Lengua y Lit. Modernas Inglesas & $10.3 \%$ & $100 \%$ & $0 \%$ \\
4 & Lengua y Lit. Modernas Francesas & $10.3 \%$ & $100 \%$ & $0 \%$ \\
5 & Lengua y Lit. Modernas Alemanas & $10.3 \%$ & $33.3 \%$ & $66.6 \%$ \\
6 & Filosofía & $10.3 \%$ & $0 \%$ & $100 \%$ \\
7 & Estudios Latinoamericanos & $6.8 \%$ & $50 \%$ & $50 \%$ \\
8 & Lengua y Lit. Modernas Portuguesas & $6.8 \%$ & $100 \%$ & $0 \%$ \\
9 & Historia & $3.4 \%$ & $100 \%$ & $0 \%$ \\
10 & Pedagogía (sUA) & $3.4 \%$ & $0 \%$ & $100 \%$ \\
11 & Desarrollo y Gestión Interculturales & $3.4 \%$ & $100 \%$ & $0 \%$ \\
12 & Lengua y Lit. Modernas Italianas & $3.4 \%$ & $100 \%$ & $0 \%$ \\
& Total & $99.9 \%$ & $62 \%$ & $38 \%$
\end{tabular}


Tabla 2. Instituciones a las que se van de movilidad los estudiantes (semestre 2017-1).

Fuente: elaboración propia a partir de los datos proporcionados por el Área de Movilidad e Intercambio Académico de la Facultad de Filosofía y Letras de la UNAM.
Con respecto a las universidades internacionales que recibieron alumnos de la FFyL destacan la Universidad Distrital Francisco José de Caldas en Colombia (10.3\%), la Université Sorbonne Nouvelle (Paris 3) en Francia (6.8\%), la Freie Universität Berlin en Alemania (6.8\%) y la Fondation Nationale Des Sciences Politiques Et Institut D'etudes Politiques De Paris (Sciences Po), en Francia (3.4\%) (ver tabla 2).

\begin{tabular}{|c|c|c|c|}
\hline No. & Institución de Educación Destino & País & Total \\
\hline 1 & Universidad Distrital Francisco José de Caldas & Colombia & $10.3 \%$ \\
\hline 2 & Freie Universität Berlin & Alemania & $6.8 \%$ \\
\hline 3 & Université Sorbonne Nouvelle (Paris 3) & Francia & $6.8 \%$ \\
\hline 4 & $\begin{array}{c}\text { Fondation Nationale Des Sciences Politiques Et Institut } \\
\text { D'etudes Politiques De Paris (Sciences Po) }\end{array}$ & Francia & $3.4 \%$ \\
\hline 5 & University College London & Reino Unido & $3.4 \%$ \\
\hline 6 & Universidad Distrital Francisco José de Caldas & Colombia & $3.4 \%$ \\
\hline 7 & Université de Montréal & Canadá & $3.4 \%$ \\
\hline 8 & Beijing Foreign Studies University (Ueeb) & China & $3.4 \%$ \\
\hline 9 & Universität Zu Köln & Alemania & $3.4 \%$ \\
\hline 10 & Universida de do Porto & Portugal & $3.4 \%$ \\
\hline 11 & Université de Montréal & Canadá & $3.4 \%$ \\
\hline 12 & Universidad Nacional de Córdoba & Argentina & $3.4 \%$ \\
\hline 13 & Universität Bremen & Alemania & $3.4 \%$ \\
\hline 14 & Universidad de Congreso & Argentina & $3.4 \%$ \\
\hline 15 & Aoyama Gakuin University & Japón & $3.4 \%$ \\
\hline 16 & $\begin{array}{l}\text { Universität Hamburg-Institut Für Geographie / } \\
\text { Facultad de Filosofía y Letras }\end{array}$ & Alemania & $3.4 \%$ \\
\hline 17 & Université de Genève & Suiza & $3.4 \%$ \\
\hline 18 & Universidad Nacional de Córdoba & Argentina & $3.4 \%$ \\
\hline 19 & Universida de de São Paulo & Brasil & $3.4 \%$ \\
\hline 20 & Universitat de Barcelona & España & $3.4 \%$ \\
\hline 21 & Universidad de Buenos Aires-Filosofía & Argentina & $3.4 \%$ \\
\hline 22 & Universidad de Buenos Aires-Geografía & Argentina & $3.4 \%$ \\
\hline 23 & King'sCollege London & Reino Unido & $3.4 \%$ \\
\hline 24 & Universidad de Groningen & Países Bajos & $3.4 \%$ \\
\hline \multirow[t]{2}{*}{25} & Universidad de Massachusetts Amherst & Estados Unidos & $3.4 \%$ \\
\hline & & Total & $99 \%$ \\
\hline
\end{tabular}

En el semestre 2017-2 (de enero a junio 2017) hubo una disminución con respecto al número de estudiantes que realizaron movilidad estudiantil. De los 29 alumnos del período anterior sólo 15 se fueron de movilidad, los cuales representan $0.19 \%$ de la población estudiantil de la entidad, lo que significa una 
Tabla 3. Porcentaje de estudiantes que realizaron movilidad internacional por licenciatura (semestre 2017-2)

Fuente: elaboración propia a partir de los datos proporcionados por el Área de Movilidad e Intercambio Académico de la Facultad de Filosofía y Letras de la UNAM.

Tabla 4. Instituciones a las que se van de movilidad los estudiantes (semestre 2017-2)

Fuente: elaboración propia a partir de los datos proporcionados por el Área de Movilidad e Intercambio Académico de la Facultad de Filosofía y Letras de la UNAM. disminución de $0.18 \%$. Entre las licenciaturas que destacan por el número de estudiantes que enviaron encontramos a las licenciaturas de Geografía y Lengua y Literaturas Modernas Inglesas con 20\% de los estudiantes cada una (ver tabla 3).

\begin{tabular}{ccccc} 
No. & Licenciatura & Total & Femenino & Masculino \\
\hline 1 & Geografía & $20 \%$ & $100 \%$ & $0 \%$ \\
2 & Lengua y Lit. Modernas Inglesas & $20 \%$ & $66.6 \%$ & $33.3 \%$ \\
3 & Filosofía & $13.3 \%$ & $100 \%$ & $0 \%$ \\
4 & Historia & $13.3 \%$ & $50 \%$ & $50 \%$ \\
5 & Lengua y Lit. Modernas Francesas & $13.3 \%$ & $50 \%$ & $50 \%$ \\
6 & Literatura Dramática y Teatro & $13.3 \%$ & $100 \%$ & $0 \%$ \\
7 & Lengua y Literaturas Hispánicas & $6.6 \%$ & $100 \%$ & $0 \%$ \\
& Total & $99.9 \%$ & $80 \%$ & $20 \%$
\end{tabular}

Con respecto a las instituciones de destino encontramos una gran diversidad (12 universidades). Las universidades que más estudiantes reciben son la National Taiwan University de China (13.3\%), la University College London de Londres (13.3\%), la University of California de Estados Unidos (13.3\%) y la King's College London (6.6\%) (ver tabla 4).

\begin{tabular}{cccc} 
No. & Institución de Educación Destino & País & Total \\
\hline 1 & National Taiwan University & China & $13.3 \%$ \\
2 & University College London & Reino Unido & $13.3 \%$ \\
3 & University of California & Estados Unidos & $13.3 \%$ \\
4 & King's College London & Reino Unido & $6.6 \%$ \\
5 & Beijing Foreign Studies University (Ueeb) & China & $6.6 \%$ \\
6 & Universida de do Porto & Portugal & $6.6 \%$ \\
7 & Universität Hamburg-Institut Für Geographie / Facultad & Alemania & $6.6 \%$ \\
& de Filosofía y Letras & & \\
8 & Université de Rouen & Francia & $6.6 \%$ \\
9 & Université Lumière Lyon 2 & Francia & $6.6 \%$ \\
10 & Université Paris 7, Paris Diderot & Francia & $6.6 \%$ \\
11 & Université Sorbonne Nouvelle (Paris 3) & Francia & $6.6 \%$ \\
12 & Fondation Nationale Des Sciences Politiques Et Institut & Francia & $3.4 \%$ \\
& D'etudes Politiques De Paris (Sciences Po) & & \\
& & Total & $99 \%$
\end{tabular}

Con base en los datos anteriores, se puede vislumbrar que para el primer semestre (2017-1) la universidad de mayor demanda es de habla hispana (Colombia), seguida por universidades de Europa. En el segundo semestre (2017-2) las universidades en el ámbito internacional que tienen mayor demanda no son de habla hispana, sino de América del Norte, Europa y Asia oriental (China y Japón). 
Tabla 5. Porcentaje de estudiantes que realizaron movilidad nacional por licenciatura (semestre 2017-1)

Fuente: elaboración propia a partir de los datos proporcionados por el Área de Movilidad e Intercambio Académico de la Facultad de Filosofía y Letras de la UNAM.

Tabla 6. Instituciones a las que se van de movilidad los estudiantes (semestre 2017-2)

Fuente: Elaboración propia a partir de los datos proporcionados por el Área de Movilidad e Intercambio Académico de la Facultad de Filosofía y Letras de la UNAM.

\section{Movilidad nacional}

En el semestre 2017-1 (de agosto a diciembre 2016), se fueron de movilidad nacional 4 estudiantes, los cuales representan $0.051 \%$ de la población estudiantil total. Como muestra la tabla 5 la licenciatura que tuvo mayor presencia en este período fue Literatura Dramática y Teatro (50\%), seguida de Pedagogía (25\%) y Desarrollo y Gestión Interculturales (25\%) (Ver tabla 5).

\begin{tabular}{ccccc} 
No. & Licenciatura & Total & Femenino & Masculino \\
\hline 1 & Literatura Dramática y Teatro & $50 \%$ & $100 \%$ & $0 \%$ \\
2 & Pedagogía & $25 \%$ & $100 \%$ & $0 \%$ \\
3 & Desarrollo y Gestión Interculturales & $25 \%$ & $100 \%$ & $0 \%$ \\
& Total & $100 \%$ & $100 \%$ & $0 \%$
\end{tabular}

Con respecto a las universidades nacionales que recibieron alumnos de la FFyL destaca la Universidad Veracruzana (75 \%) (ver tabla 6).

\begin{tabular}{ccc} 
No. & Institución de Educación Superior Destino & Total \\
\hline 1 & Universidad Veracruzana & $75 \%$ \\
2 & Universidad Autónoma del Estado de Hidalgo & $25 \%$ \\
& Total & $100 \%$
\end{tabular}

\section{Implicaciones}

Al tener la experiencia de ir de movilidad al extranjero, el estudiante se ve inmerso en un ambiente diferente al que conoce en el ámbito cultural, económico, político y social. Un factor importante es el manejo del idioma, pues, de no dominarse en un grado suficiente, dificulta de manera significativa la integración y el aprovechamiento del estudiante, tanto en lo académico como en la experiencia de vida (López, 2010). Por otro lado, las oportunidades y beneficios que implica la movilidad estudiantil son un factor sobresaliente del proceso. Al respecto, Martínez (2004, p. 235) plantea que entre los beneficios están "las relaciones de estudiantes y profesores de diferentes culturas y en contextos diversos, [que] a través de una movilidad bien informada y organizada permite experiencias globales pedagógicas ampliando el espacio pedagógico local e intercambiando prácticas e ideas vividas en espacios sociales plurales".

\section{Consideraciones finales}

Los estudiantes que realizan movilidad representan un pequeño porcentaje respecto a la población estudiantil total de la Facultad de Filosofía y Letras de la unAm. La tendencia de la movilidad en esta entidad es hacia el ámbito 
internacional, ya que la mayoría de los estudiantes la realizan en universidades internacionales y muy pocos en nacionales, incluso en el segundo período se abandonó por completo esta modalidad.

Otro punto importante es que, en la mayoría de los casos, la movilidad estudiantil la realizan más mujeres que hombres. Asimismo, un aspecto a resaltar es que se lleva a cabo principalmente en países donde no se habla castellano, sino en instituciones de la Unión Europea, América del Norte y Asia oriental, lo cual permite vislumbrar que los estudiantes están eligiendo mayormente países donde se habla un idioma distinto a su lengua materna.

Cabe destacar la importancia que tiene la movilidad en la vida del estudiante ya que constituye una alternativa para elevar la calidad académica, por tanto, fortalece su formación integral, al otorgar la oportunidad de compartir experiencias, intercambiar conocimiento y mantenerse actualizados. En el caso de la movilidad nacional, se establece contacto con otras instituciones de educación superior (IES) para el desarrollo en diversas áreas el conocimiento; con ello se complemente la formación profesional. Por todo lo anterior es importante fomentar la movilidad estudiantil en las universidades y hacer más difusión acerca de los diversos programas que existen en las instituciones. Asimismo, se debe monitorear a los estudiantes en su formación superior y comprobar que una experiencia temprana de viaje académico en el nivel medio superior fortalece su preparación académica.

\section{Referencias}

* Corbella, V. y Elías, S. (2018). Movilidad estudiantil universitaria: ¿qué factores inciden en la decisión de elegir Argentina como destino? Perfiles educativos, 40(160), 120-140. Recuperado de: http://www.scielo.org.mx/pdf/peredu/v40n160/01852698-peredu-40-160-120.pdf Consultado el 08/12/2018.

* Espacio Común de Educación Superior (ECOES). (2019). Espacio Común de Educación Superior. Recuperado de: http://www.ecoes.unam.mx/presentacion.html.

* Facultad de Filosofía y Letras. (2019). Programas académicos. Recuperado de: http://www.filos.unam.mx/index.php/programas academicos/.

* Fraile, A. (2006). El sistema universitario europeo como modelo posible para la educación superior latinoamericana. Revista Electrónica de Investigación Educativa 8(1), 1-15. Recuperado de: http://www.scielo.org.mx/pdf/redie/v8n1/v8n1a3.pdf.

- López, M. T. (2010). El intercambio estudiantil como recurso promotor del desarrollo humano. (tesis de maestría). Recuperado de: http://www.bib.uia.mx/tesis/ pdf/015328/015328.pdf.

- Martínez, J. B. (2004). Movilidad/movilización de profesorado y estudiantes para la formación. Revista Interuniversitaria de Formación del Profesorado, 18(3), 233250. Recuperado de: https://www.redalyc.org/articulo.oa?id=27418315. 
* Rodríguez Gómez, R. (2018). Dos décadas del proceso de Bolonia. Revista Mexicana de Investigación Educativa, 23(76), 7-14. Recuperado de: http://www.scielo.org.mx/ $\mathrm{pdf} / \mathrm{rmie} / \mathrm{v} 23 \mathrm{n} 76 / 1405-6666-\mathrm{rmie}-23-76-7 . \mathrm{pdf}$.

* Secretaría de Educación Pública (SEP). (2007). Programa Sectorial de Educación 2007-2012. México: SEP.

* Secretaría de Educación Pública (SEP). (2013). Programa Sectorial de Educación 2013-2018. México: SEP.

* UNAM-Dirección General de Comunicación Social (2018). Intercambio de alumnos de la UNAM con universidades de 29 países. Recuperado de: http://www.dgcs.unam. $\mathrm{mx/boletin/bdboletin/2018} \mathrm{814.html}$.

* UNAM-Dirección General de Cooperación e Internacionalización (2018). Lineamientos para regularla movilidad estudiantil de Licenciatura en la Universidad Nacional Autónoma México. Recuperado de: http://www.abogadogeneral.unam. $\mathrm{mx} /$ legislacion/abogen/documento.html?doc id=96.

\section{Cómo citar este artículo}

* Sandoval Rosete, Luis Alberto y Santiago Munilla, Vianey (2019). Explorar la movilidad estudiantil: Facultad de Filosofía y Letras de la UNAM. Revista Digital Universitaria (RDU). Vol. 20, núm. 5 septiembre-octubre. Dol: http://doi.org/10.22201/ codeic.16076079e.2019.v20n5.a9.

Recepción: 27/03/2019. Aprobación: 27/06/2019. 